\title{
Identification of Transient Temperature Distribution in a Thick-Walled Component
}

\author{
Piotr Duda, Mariusz Konieczny \\ Institute of Thermal and Process Engineering, Department of Mechanical Engineering, Cracow University of Technology \\ Al. Jana Pawła II 37, 31-864 Kraków, Poland \\ pduda@mech.pk.edu.pl; mk.mk@wp.pl
}

\begin{abstract}
The paper presents a method of transient temperature estimation in a thick-walled pressure component. Despite the unknown boundary condition on the internal surface of the component, the method allows to determine the temperature distribution thanks to "measured" temperature histories determined in easily accessible points located on the component outer surface. On the outer component surface, heat exchange by radiation and convection is assumed. The proposed method is verified numerically. The presented method for controlling temperature is also suitable for nuclear power plants because it does not require drilling holes for sensors in the pressure element walls.
\end{abstract}

Keywords: inverse problems, heat conduction, control volume method, diagnostic system

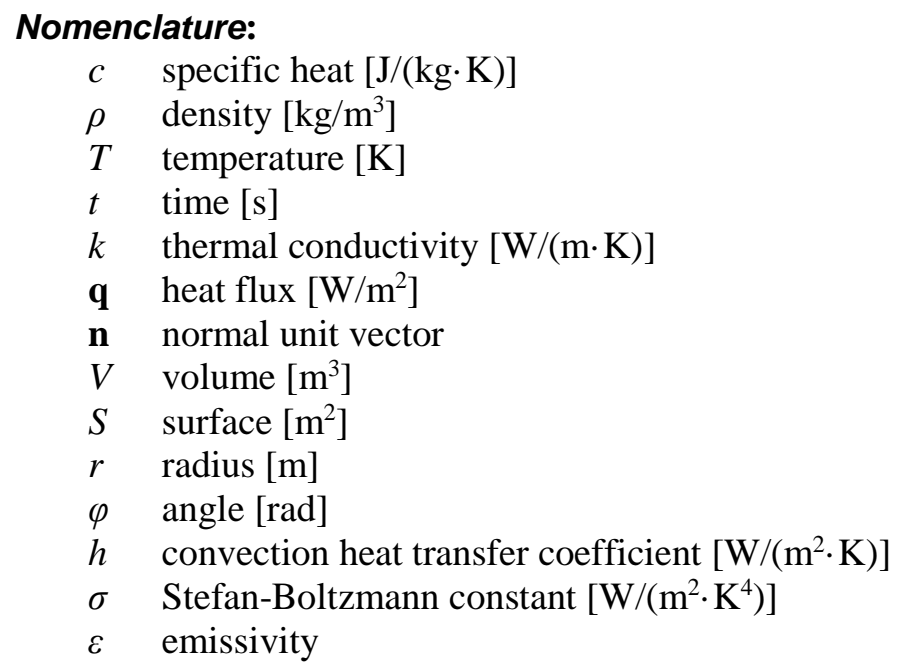

\section{Introduction}

The heat transfer coefficient (HTC) is an important parameter for heat exchanger design [1]. Automatic transmission fluid (ATF) and water based aluminum oxide $\left(\mathrm{Al}_{2} \mathrm{O}_{3}\right)$ nanofluids are used in a multiport slab minichannel heat exchanger under laminar flow conditions. A lower improvement of HTC is observed for lower volume fraction; however, the significant enhancement of HTC is observed for higher volume fraction. Heat fluxes modeling of combustion chamber is presented in [2]. Prediction of convective HTC is based on empirical correlations [3]. The accuracy of HTC, which is estimated in this way is not high. On the other hand, analysing the phenomena that take place in the flowing fluid numerically [1] is very time-consuming. Another way to determine the temperature distribution without the need to approximate HTC is finding a solution of the inverse heat conduction problem (IHCP) in the device under analysis [4-7].

The paper presents a method of transient temperature estimation in a thick-walled pressure component. Despite the unknown boundary condition on the internal surface of the component, the method allows to determine the temperature distribution thanks to "measured" temperature histories determined in easily accessible points located on the component outer surface. On the outer component surface, heat exchange by radiation and convection is assumed. The proposed 
method is verified numerically. The presented method for controlling temperature is also suitable for nuclear power plants because it does not require drilling holes for sensors in the pressure element walls.

\section{Formulation of the method}

The equation governing the transient-state heat conduction problem has the following form:

$$
c(T) \rho(T) \frac{\partial T}{\partial t}=-\nabla \cdot \mathbf{q}
$$

where $\mathbf{q}$ is the heat flux vector. Fourier's law for an isotropic material takes the following form:

$$
\mathbf{q}=-k \nabla T
$$

All material properties ( $c$ - specific heat, $\rho$ - density, $k$ - thermal conductivity) are assumed as known functions of temperature. The control volume method is used and equation (1) is integrated over control volume $V$ with bounding surface $S$. By applying the mean value theorem and the divergence theorem the following equation can be written

$$
V c(\bar{T}) \rho(\bar{T}) \frac{d \bar{T}}{d t}=-\oint_{S} \mathbf{q} \cdot \mathbf{n} d S
$$

where the bar indicates an average value in volume $V$ and $\mathbf{n}$ is a normal unit surface vector directed to the outside of the control volume. If temperature does not vary in a cylindrical component along the generatrix but changes versus the radius and along the circumference, the temperature distribution is two-dimensional $T(r, \varphi, t)$. Additionally, the problem is assumed to be symmetrical with respect to the vertical surface.

For such a case, the analysis can be carried out only for a cross-section, which is presented in Fig. 1. It is divided into 52 control volumes. Assuming that the boundary condition on the component outer surface is known and an unknown boundary condition occurs on the inner surface, the inverse problem may be solved starting with a heat balance equation for the control volume associated with node $i=40$.

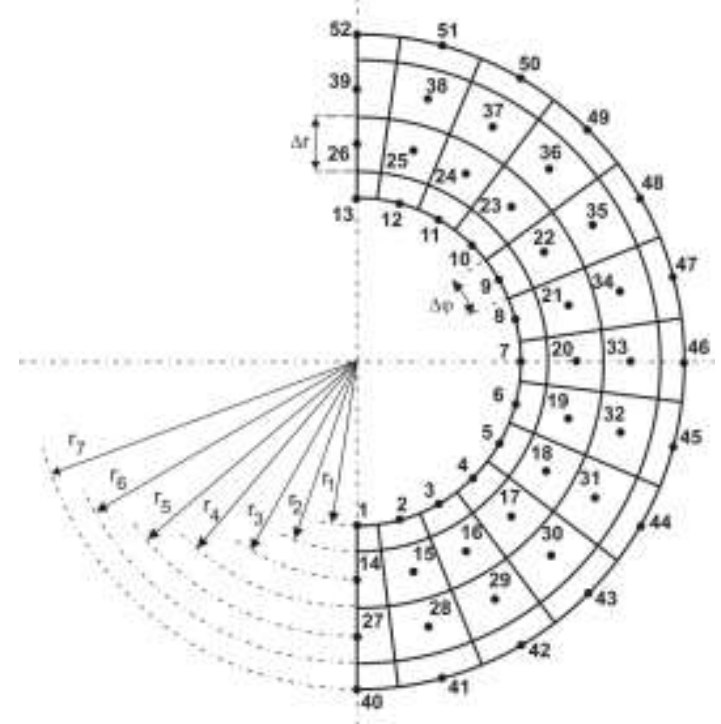

Fig. 1: Division of a cylindrical component cross-section into control volumes.

The equation governing the transient-state heat conduction problem has the following form: 


$$
\frac{d T_{i}}{d t}=\frac{4}{c_{i} \rho_{i}\left(r_{7}^{2}-r_{6}^{2}\right) \cdot \Delta \varphi}\left[\frac{k_{i+1}+k_{i}}{2} \cdot \frac{0.5 \cdot \Delta r}{r_{7} \cdot \Delta \varphi}\left(T_{i+1}-T_{i}\right)+\frac{k_{i-13}+k_{i}}{2} \cdot \frac{r_{6} \cdot 0.5 \cdot \Delta \varphi}{\Delta r}\left(T_{i-13}-T_{i}\right)+\text { con }_{\text {out }}+\text { rad }_{\text {out }}\right]
$$

where $i=40, \quad k_{i}=k\left(T_{i}\right), \quad c_{i}=c\left(T_{i}\right), \quad c_{i}=c\left(T_{i}\right), \quad \rho_{i}=\rho\left(T_{i}\right)$. The terms con $_{\text {out }}$, rad $_{\text {out }}$ are the heat rates, which are transferred to the analysed control volume from the environment by convection and radiation, respectively. They can be expressed by the following equations:

$$
\begin{aligned}
& \operatorname{con}_{\text {out }}=h\left(T_{\text {OUT }}-T_{i}\right) \cdot r_{7} \cdot 0.5 \cdot \Delta \varphi \\
& \operatorname{rad}_{\text {out }}=\varepsilon \sigma\left(\left(T_{\text {OUT }}+T_{0}\right)^{4}-\left(T_{i}+T_{0}\right)^{4}\right) \cdot r_{7} \cdot 0.5 \cdot \Delta \varphi
\end{aligned}
$$

where $h$ is convection heat transfer coefficient, $\varepsilon$ is the emissivity of the surface and $\sigma=5.67 \times 10^{-8} \mathrm{~W} / \mathrm{m}^{2} \cdot \mathrm{K}^{4}$ is the Stefan-Boltzmann constant. For pipes, which are perfectly insulated on the outer surface, both the terms defined in (5) are equal to zero and can be omitted.

The heat balance equations for control volumes $i=41-51$ are expressed as:

$$
\begin{aligned}
& \frac{d T_{i}}{d t}=\frac{2}{c_{i} \rho_{i}\left(r_{7}^{2}-r_{6}^{2}\right) \cdot \Delta \varphi}\left[\frac{k_{i-1}+k_{i}}{2} \cdot \frac{0.5 \cdot \Delta r}{r_{7} \cdot \Delta \varphi}\left(T_{i-1}-T_{i}\right)+\frac{k_{i-13}+k_{i}}{2} \cdot \frac{r_{6} \cdot \Delta \varphi}{\Delta r}\left(T_{i-13}-T_{i}\right)+\right. \\
& \left.+\frac{k_{i+1}+k_{i}}{2} \cdot \frac{0.5 \cdot \Delta r}{r_{7} \cdot \Delta \varphi}\left(T_{i+1}-T_{i}\right)+h\left(T_{\text {OUT }}-T_{i}\right) \cdot r_{7} \cdot \Delta \varphi+\varepsilon \sigma\left(\left(T_{\text {OUT }}+T_{0}\right)^{4}-\left(T_{i}+T_{0}\right)^{4}\right) \cdot r_{7} \cdot \Delta \varphi\right]
\end{aligned}
$$

and for the control volume, which is associated with node $i=52$ the following can be written:

$$
\begin{aligned}
& \frac{d T_{i}}{d t}=\frac{4}{c_{i} \rho_{i}\left(r_{7}^{2}-r_{6}^{2}\right) \cdot \Delta \varphi}\left[\frac{k_{i-1}+k_{i}}{2} \cdot \frac{0.5 \cdot \Delta r}{r_{7} \cdot \Delta \varphi}\left(T_{i-1}-T_{i}\right)+\frac{k_{i-13}+k_{i}}{2} \cdot \frac{r_{6} \cdot 0.5 \cdot \Delta \varphi}{\Delta r}\left(T_{i-13}-T_{i}\right)+\right. \\
& \left.+h\left(T_{\text {OUT }}-T_{i}\right) \cdot r_{7} \cdot 0.5 \cdot \Delta \varphi+\varepsilon \sigma\left(\left(T_{\text {OUT }}+T_{0}\right)^{4}-\left(T_{i}+T_{0}\right)^{4}\right) \cdot r_{7} \cdot 0.5 \cdot \Delta \varphi\right]
\end{aligned}
$$

In order to solve the inverse problem the temperature transients in nodes $i=40-52$ are measured. Based on these data temperature derivatives versus time can also be calculated. If the boundary conditions on the outer surface are known and the material properties do not depend on the temperature, the only unknown variable in eq. (4) is $T_{i-13}=T_{27}$. Similarly, other temperatures $\mathrm{T}_{28}-\mathrm{T}_{39}$ can be determined from equations (6-7). If the material properties $c, \rho$ and $k$ are temperaturedependent, the appropriate equation can be solved iteratively.

Once the temperature values in nodes 27 to 39 are obtained, an inverse solution can be run again to find the temperatures in points 14 to 26 by writing the heat balance equations for the control volumes associated with nodes 27 to 39. A similar system of equations can be written for volumes 14 to 26 to calculate temperature histories from nodes 1 to 13.

Solving inverse problems is more difficult compared to direct ones because inverse problems are ill-posed. There are many methods to solve this problem. In this work, smoothing filters are used. The filters are based on local polynomial approximation ( $3^{\text {rd }}$ order kind and eleven subsequent measurement points) and they smooth out the measured temperature curves [7].

\section{Numerical verification}

The accuracy of the inverse method is presented in numerical tests and conducted for the thick-walled pipe, as shown in Fig. 2. The pipe is made of steel St41K, its outer diameter and the wall thickness is $219 \mathrm{~mm}$ and $40 \mathrm{~mm}$, respectively. It is assumed that the pipe with the initial uniform temperature of $T_{0}=20^{\circ} \mathrm{C}$ is partially flooded by the medium. The medium temperature is constant $T_{m}=200{ }^{\circ} \mathrm{C}$ and the heat transfer coefficient is $H T C_{2}=400 \mathrm{~W} / \mathrm{m}^{2} \mathrm{~K}$. The upper part of the inner 
surface is surrounded by air at constant temperature $T_{\text {air }}=20^{\circ} \mathrm{C}$ and the coefficient for the convection is $H T C_{1}=5 \mathrm{~W} / \mathrm{m}^{2} \mathrm{~K}$. On the outer surface, both natural convection and radiation occur simultaneously. The ambient temperature of the air is $T_{\text {air }}$ $=20^{\circ} \mathrm{C}$ and the mean value of the heat transfer coefficient for a pipe is calculated according to the equation (8).

$$
h=5 \cdot \sqrt{\frac{T_{\text {out }}-\bar{T}_{40-52}}{T_{\text {out }} \cdot d_{\text {out }}}}
$$

Where $\bar{T}_{40-52}$ is the mean temperature of nodes 40-52 in all time points. In this case the temperature $\bar{T}_{40-52}=74.4^{\circ} \mathrm{C}$ and the calculated value of $h$ for the natural convection is $4.60 \mathrm{~W} / \mathrm{m}^{2} \mathrm{~K}$. The emissivity of the pipe outer surface is assumed as 0.8 .

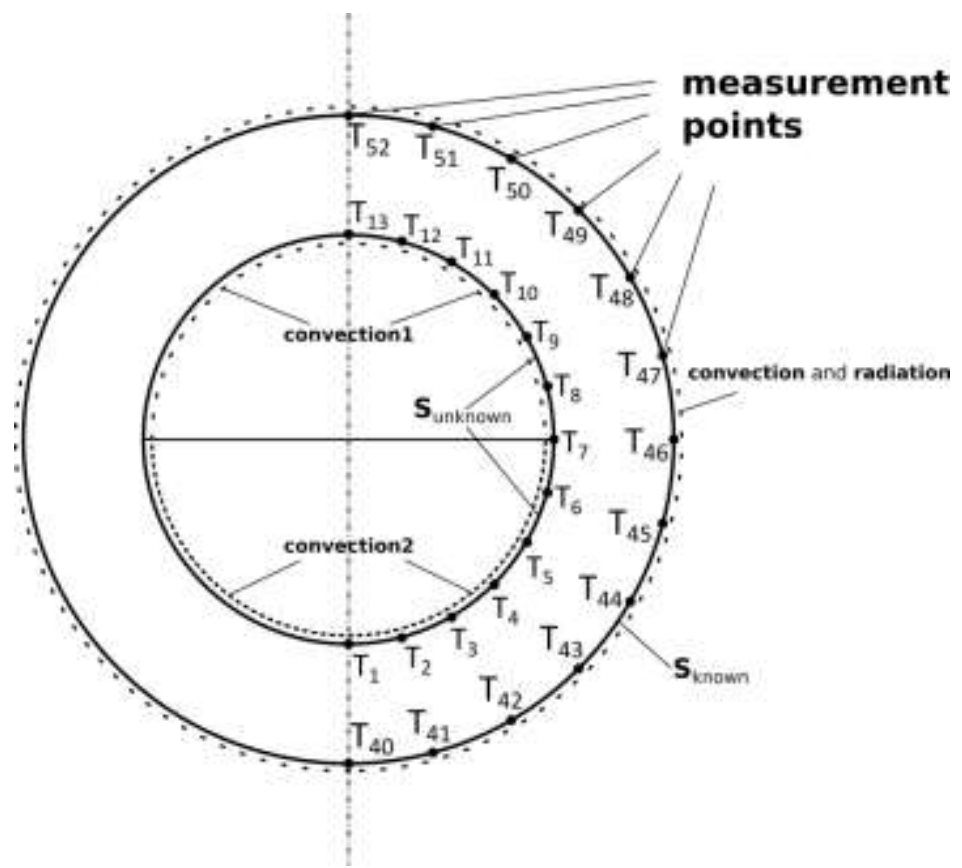

Fig. 2: The cross section of the pipe.

The temperature transients at nodes 40-52 are obtained from a direct solution by means of ANSYS software. The region is divided into four node quadrilateral finite elements. The mesh is built with 15 elements across the wall thickness and 120 along the circumference. The whole temperature field in the analyzed area is assumed as "measurement data" for the inverse solution. In order to bring the numerical test closer to real conditions, "noisy measured data" are obtained by adding normal random errors to "precise data". The generated error average value is equal to zero for all kinds of disturbed input data. The "noisy measured data" are used during inverse solution. The "measured" noisy temperatures transients in nodes $40-52$ (for error $= \pm 0.5^{\circ} \mathrm{C}$ ) and the fluid temperature are shown in Fig. 3. 


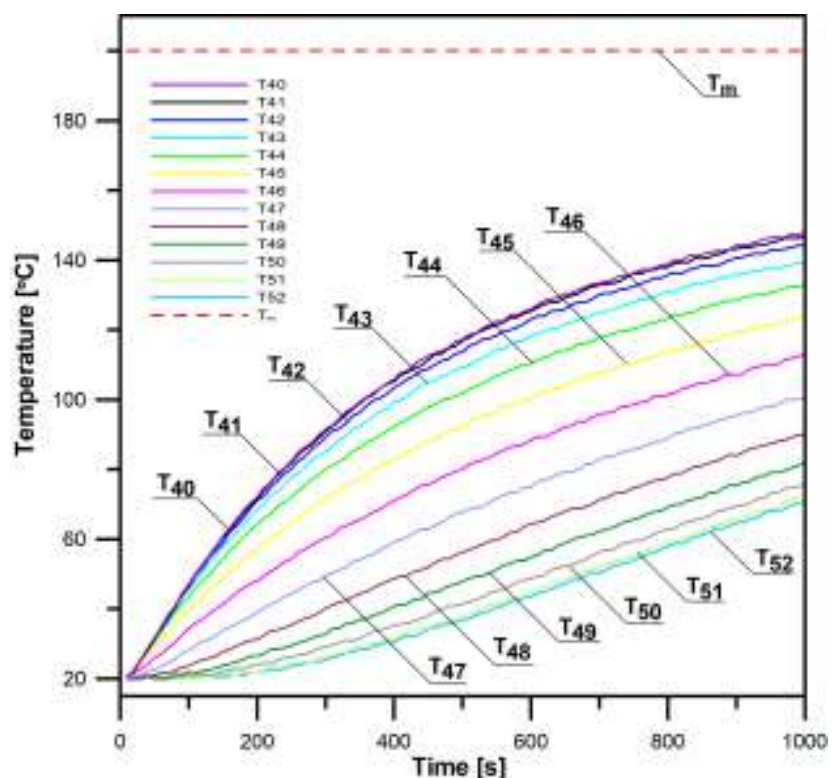

Fig. 3: "Measured" temperature transients in nodes 40-52.

The purpose of the proposed inverse method is to identify the transient temperature distribution in the whole cross section of the pipe based on the known boundary condition on the outer surface with unknown boundary condition on the inner surface of the pipe. The algorithm calculates firstly temperature transients at 27-39 nodes by solving 13 heat balance equations for control volumes which are associated with nodes 40-52 using Eq. (4-7). In the same way, one calculates temperatures 14-26 and 1-13. The results' accuracy in time and space can be seen in Figs. 4 and 5, where comparisons between the inverse method results and the exact values from the direct method are presented.

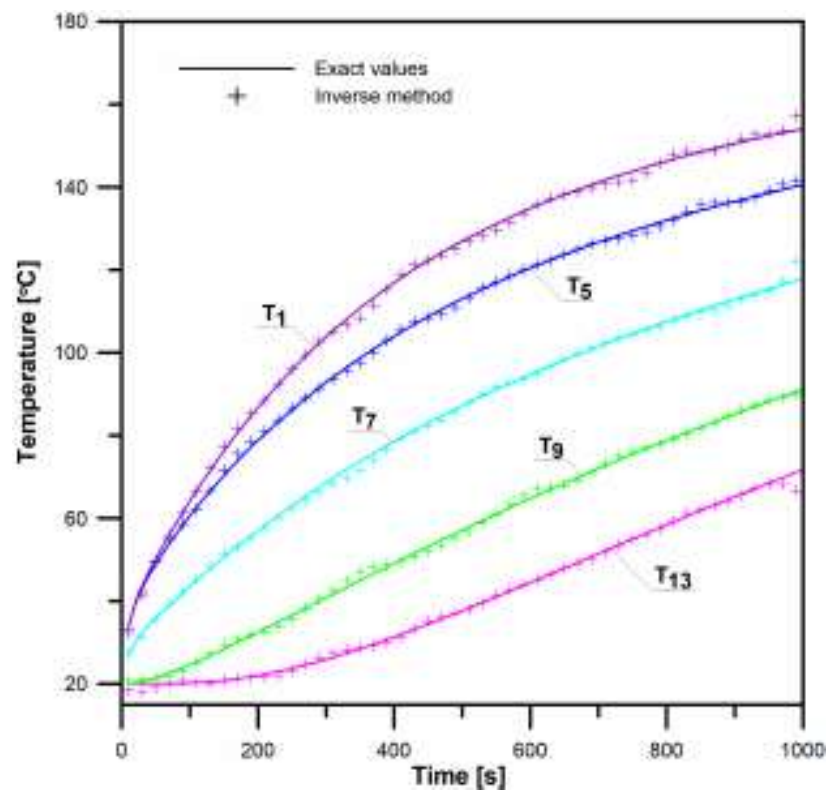

Fig. 4: Comparison between exact temperature transients and those calculated by the inverse method on the pipe inner surface in selected nodes. 


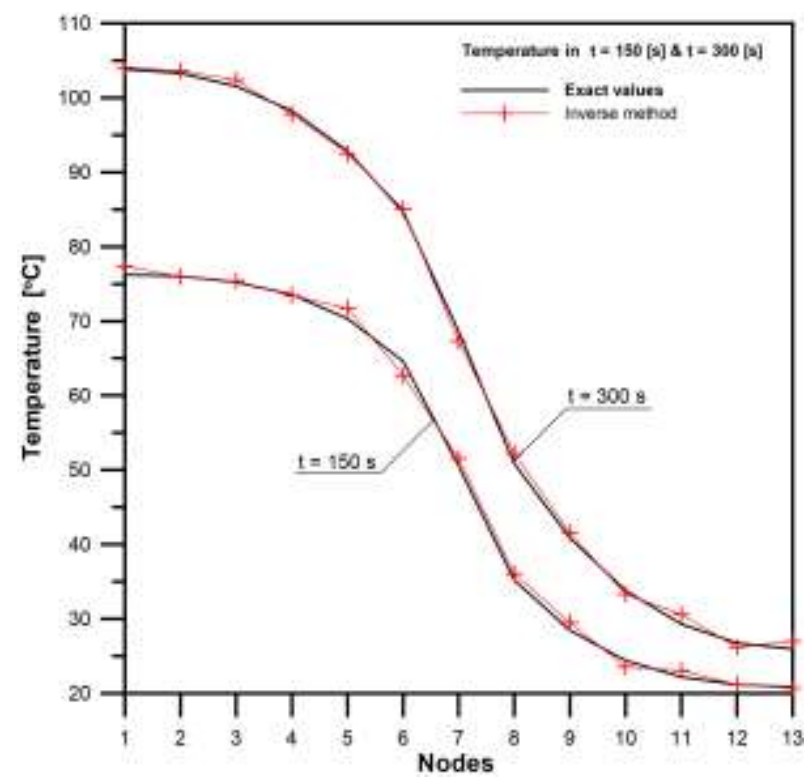

Fig. 5: Comparison between exact temperature $\mathrm{T}_{1}-\mathrm{T}_{13}$ and inverse temperatures at chosen time of $150 \mathrm{~s}$ and $300 \mathrm{~s}$.

In order to reduce the oscillations, the data is "smoothed out" using digital filters, which are based on the local polynomial approximation. Eleven subsequent auxiliary points are approximated locally by means of the third grade polynomial. Using the polynomial of the $3^{\text {rd }}$ order and eleven subsequent measurement points, the formula for derivative $d T / d t$ is obtained. The results are obtained using the time step of $\Delta t=10 \mathrm{~s}$.

If the outer surface $S_{\text {known }}$ is not insulated it is exposed to convection and radiation heat transfer. Both the heat fluxes are calculated numerically. Their mean values on the outer pipe surface versus time are shown in Fig. 6 . The larger part of the heat is transmitted by radiation and it increases with the rise of pipe temperature.

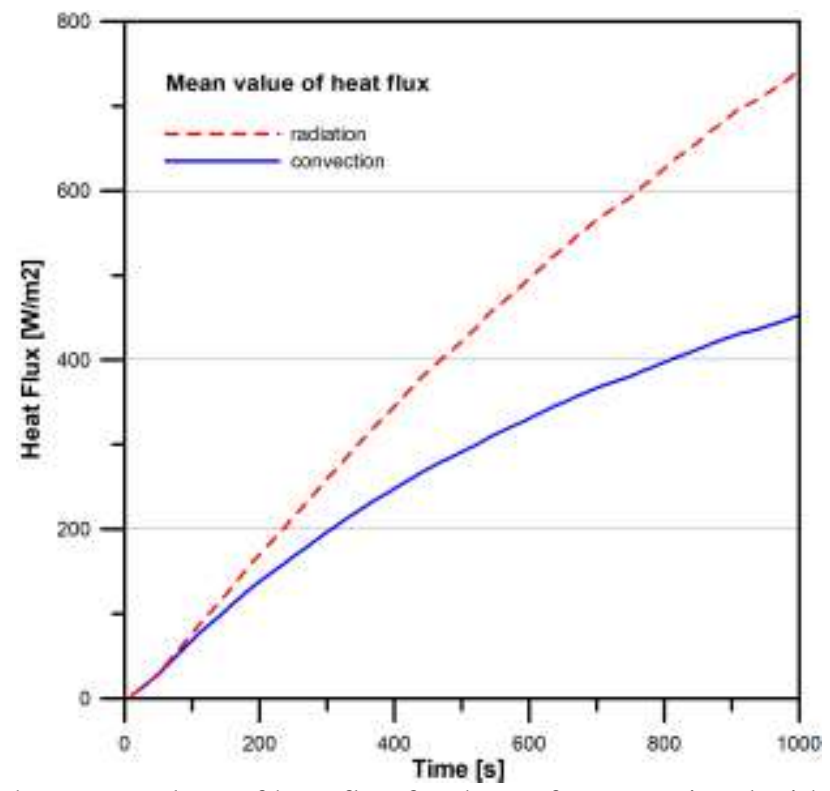

Fig. 6: The mean values of heat flux for the surface associated with nodes 40-52.

The total temperature errors are the effect of the measurement uncertainties and the method ill conditioning. The part due to the ill conditioning can be found from the solution obtained based on "undisturbed measurement data". The measurement uncertainties are usually the main reason of total errors. 


\section{Conclusion}

The paper presents a method of transient temperature estimation in a thick-walled pressure component. Despite the unknown boundary condition on the internal surface of the component, the method allows to determine the temperature distribution thanks to "measured" temperature histories determined in easily accessible points located on the component outer surface. On the outer component surface heat exchange by radiation and convection is assumed. The larger part of the heat is transmitted by radiation and it increases with the rise of pipe temperature. The proposed method applicability is shown through a comparison with the results obtained from the direct solution. The presented numerical example demonstrates high stability and accuracy of the inverse method for the "noisy measured data". The presented method for controlling temperature is also suitable for nuclear power plants because it does not require drilling holes for sensors in the pressure element walls.

\section{Acknowledgements}

This research was financed by the National Science Centre, Poland, UMO-2015/19/B/ST8/00958. The author declares that there is no conflict of interest regarding the publication of this paper.

\section{References}

[1] M. Ismail, S. Fotowat, A. Fartaj, "Numerical Simulation of $\mathrm{Al}_{2} \mathrm{O}_{3} /$ Automatic Transmission Fluid and $\mathrm{Al}_{2} \mathrm{O}_{3} / \mathrm{Water}$ Nanofluids in a Compact Heat Exchanger," Journal of Fluid Flow, Heat and Mass Transfer, vol. 3, pp. 34-43, 2016.

[2] A. Adami1, M. Mortazavi1, M. Nosratollahi, "Heat Transfer Modeling of Bipropellant Thrusters for using in Multidisciplinary Design Optimization Algorithm," Journal of Fluid Flow, Heat and Mass Transfer, vol. 2, pp. 40-46, 2015.

[3] D. R. Bartz, "Turbulent boundary-layer heat transfer from rapidly accelerating Wow of rocket combustion gases and of heated air," Jet Propulsion Laboratory, California, Tech. Rep. NASA-CR-62615, 1963.

[4] O.M. Alifanov, and A.V. Nenarokomov, "Three-dimensional boundary inverse heat conduction problem for regular coordinate systems", Inverse Probl. Eng., vol. 7, no. 4, pp. 335-362, 1999.

[5] P. Duda, "Solution of an inverse axisymmetric heat conduction problem in complicated geometry," Int. J. Heat Mass Transf., vol. 82, pp. 419-428, 2015.

[6] P. Duda, "A method for transient thermal load estimation and its application to identification of aerodynamic heating on atmospheric reentry capsule," Aerosp. Sci. Technol., vol. 51, pp. 26-33, 2016.

[7] P. Duda, and M. Konieczny, "A new algorithm for solving an inverse transient heat conduction problem by dividing a complex domain into parts," Int. J. Heat Mass Transf., vol. 128, pp. 865-874, 2019. 